\title{
BMJ Open Delivering the right information to the right person at the right time to facilitate deprescribing in hospital: a mixed methods multisite study to inform decision support design in Australia
}

\author{
Melissa T Baysari (D , ,,2 Mai Duong, ${ }^{3}$ Wu Yi Zheng, ${ }^{1,2}$ Amy Nguyen, ${ }^{2,4}$ Sarita Lo, ${ }^{5}$ \\ Brendan Ng, ${ }^{6}$ Angus Ritchie, ${ }^{7,8}$ David Le Couteur, ${ }^{9}$ Andrew McLachlan, ${ }^{10}$ \\ Alexandra Bennett, ${ }^{11}$ Sarah Hilmer ${ }^{3,12}$
}

To cite: Baysari MT, Duong M, Zheng WY, et al. Delivering the right information to the right person at the right time to facilitate deprescribing in hospital: a mixed methods multisite study to inform decision support design in Australia. BMJ Open 2019;9:e030950. doi:10.1136/ bmjopen-2019-030950

- Prepublication history and additional material for this paper are available online. To view these files, please visit the journal online(http://dx.doi. org/10.1136/bmjopen-2019030950)

Received 8 April 2019 Revised 20 May 2019 Accepted 11 June 2019

Check for updates

(C) Author(s) (or their employer(s)) 2019. Re-use permitted under CC BY-NC. No commercial re-use. See rights and permissions. Published by BMJ.

For numbered affiliations see end of article.

Correspondence to

Dr Melissa T Baysari;

melissa.baysari@sydney.edu.au

\section{ABSTRACT}

Objectives To inform the design of electronic decision support (EDS) to facilitate deprescribing in hospitals we set out to (1) explore the current processes of in-hospital medicines review, deprescribing and communication of deprescribing decisions with the patient's general practitioner (GP), (2) identify barriers to undertaking these tasks and (3) determine user preferences for EDS.

Design Multimethod, multisite study comprising observations, semistructured interviews and focus groups. Setting General medicine, geriatric medicine and rehabilitation wards at six hospitals in two local health districts in Sydney, Australia and primary care practices in one primary healthcare district in Sydney, Australia. Participants 149 participants took part in observations, interviews and focus groups, including 69 hospital doctors, 13 nurses, 55 pharmacists and 12 GPs.

Main outcome measures Observational data on who was involved in medicines review and deprescribing, when medicines review took place, and what artefacts (eg, forms) were used. Participants reported perceptions of medicines review, polypharmacy and deprescribing and preferences for EDS.

Results Deprescribing, undertaken during medicines review, was typically performed by a junior doctor, following a decision to deprescribe by a senior doctor. Key barriers to deprescribing included a perception that deprescribing was not the responsibility of hospital doctors, a lack of confidence among junior doctors and pharmacists in broaching this topic with senior doctors and a lack of patient engagement in the deprescribing process. In designing EDS, the tools, likely to be used by junior doctors, pharmacists and nurses, should be available throughout the hospitalisation and should comprise noninterruptive evidence-based guidance on why and how to deprescribe.

Conclusions Deprescribing decisions are complex and influenced by multiple factors. The implementation of EDS alone is unlikely to address all barriers identified. To achieve sustained improvements in monitoring of polypharmacy and subsequent deprescribing, a multifaceted intervention is needed.

\section{Strengths and limitations of this study}

- This multimethod, multisite study comprised an in-depth investigation of medicines review and deprescribing with a large number of multidisciplinary clinicians.

By complementing interviews with in situ observation, we were able to validate some participants' perceptions with objective data.

- Our results are primarily drawn from interviews and may be subject to biases associated with self-report.

- The perceptions and practices described may not be generalisable to other clinical services or different settings.

\section{INTRODUCTION}

Potentially inappropriate polypharmacy occurs in approximately half of older hospitalised patients internationally and is not usually addressed during routine hospital care. ${ }^{2}$ Addressing inappropriate polypharmacy in older inpatients is essential to meet the Third WHO Global Patient Safety Challenge: 'Medication Without Harm', which targets high risk situations, polypharmacy and transitions of care, aiming to reduce avoidable harm related to medications by $50 \%$ over 5 years. ${ }^{3}$ The hospital setting provides an opportunity for undertaking comprehensive medication reviews, one possible outcome of which is deprescribing. Deprescribing, defined as the cessation of a potentially inappropriate medication, supervised by a healthcare professional with the goal of managing polypharmacy and improving health outcomes, ${ }^{4}$ is gaining momentum internationally. ${ }^{4-6}$

As hospitals are increasingly transitioning from paper-based medication charts to 
electronic order entry systems, an opportunity exists to integrate decision support and guidance for medication review and deprescribing into these systems. There is now good evidence to show that when well-designed and targeted, electronic decision support (EDS) can have significant impacts on care outcomes. ${ }^{7-9}$ This is applicable to decision support targeting potentially inappropriate prescribing, with two recent reviews determining that computerised interventions are effective in reducing potentially inappropriate prescribing in hospitalised patients. ${ }^{1011}$ However, there are also an increasing number of studies demonstrating that decision support is ignored or not used by clinicians. ${ }^{12}{ }^{13}$ In fact, both above-mentioned reviews highlighted this problem and stressed the importance of working with users to ensure that decision support is relevant and aligns well with clinician workflow. ${ }^{1011}$

Small-scale, single-site studies have explored provider awareness and barriers to medication review and deprescribing ${ }^{14-16}$ but an in-depth understanding of current processes and barriers across different provider groups and settings is required in order to design EDS for hospital practice to align well with the workflow of all users. EDS can facilitate decision making if it provides the right information at the right time to the right person. ${ }^{9}$ However, determining the right information and identifying the right time and person to target are challenging. The aim of this study was to explore the current processes of in-hospital medicines review, deprescribing and communication of deprescribing with the patient's general practitioner (GP). In particular, we aimed to identify barriers to undertaking these tasks, and to explore user preferences for decision support, in order to inform the design of EDS to support these key processes.

\section{METHOD}

A multimethods approach was used comprising observations of clinicians (doctors and pharmacists) and interviews and focus groups with clinicians (hospital doctors, nurses, pharmacists and GPs).

\section{Setting and participants}

This study was undertaken at six hospitals in two local health districts and in primary care practices in one primary healthcare district in Sydney, Australia. These districts were selected because they were located in different socioeconomic regions in Sydney. Small, medium and large hospitals in each region were included as study sites. See supplementary file for additional information on study context.

Table 1 outlines details of the study sites at the time of data collection and the participants who took part. All hospitals used a commercial clinical information system, PowerChart (current code level 2015, Cerner Corporation, Kansas City, Missouri, USA: https://www.cerner. com), but only one hospital used electronic medication management at the time of data collection.
To recruit hospital participants, researchers attended existing education sessions and ward meetings to deliver brief presentations on the project, and posters were displayed on wards. Relevant clinical leads were also informed of the study and asked to direct interested participants to researchers. To recruit GPs, advertisements were sent out through the local primary care health network promotional channels and interested participants contacted the researcher directly.

In total, 149 clinicians participated in the study across six hospitals and the community (69 hospital doctors, 55 pharmacists, 13 nurses and 12 GPs). The study included hospital-based doctors, nurses and pharmacists who work in geriatric medicine, general medicine and rehabilitation wards at each site. Approximately 52 hours of observation were undertaken across four hospitals (table 1 and supplementary file).

\section{Data collection}

\section{Observations of hospital clinicians}

Researchers (two pharmacists and a geriatrician) shadowed hospital clinicians as they completed routine tasks related to medicines review and collected detailed handwritten notes on the following: who was involved in medicines review and deprescribing, where medicines review took place and what artefacts (eg, forms, electronic systems) were used. Researchers also classified the type of medicines review that they observed into either (1) an initial review at admission to hospital, (2) a review during a patient's stay (ie, follow-up) or (3) a review at discharge from hospital.

\section{Interviews and focus groups with hospital-based clinicians and} GPS

Semistructured interviews and focus groups with hospital clinicians focused on four main areas: the current process of medicines review, polypharmacy, deprescribing and preferences for EDS. Interview questions are presented in the supplementary file. Semistructured interviews and focus group with GPs in primary care practices focused on four main areas: perceptions of the current process of medicines review in hospital, polypharmacy, deprescribing and preferences for communication of in-hospital deprescribing on discharge to improve continuity of medication management after review in hospital.

\section{Data analysis}

Handwritten notes were taken during observations and were collated to enable identification of key elements of medicines review and deprescribing.

Interviews and focus groups were audio-recorded, transcribed verbatim and de-identified. An iterative general inductive approach was used for analysis. ${ }^{17}$ Three researchers independently reviewed de-identified transcripts and coded the data. The four focus areas (medicines review, polypharmacy, deprescribing and EDS) guided initial coding of the transcripts. Multidisciplinary researchers, including clinicians, EDS specialists and 
Table 1 Study site information and details of interviews, focus groups and observations undertaken

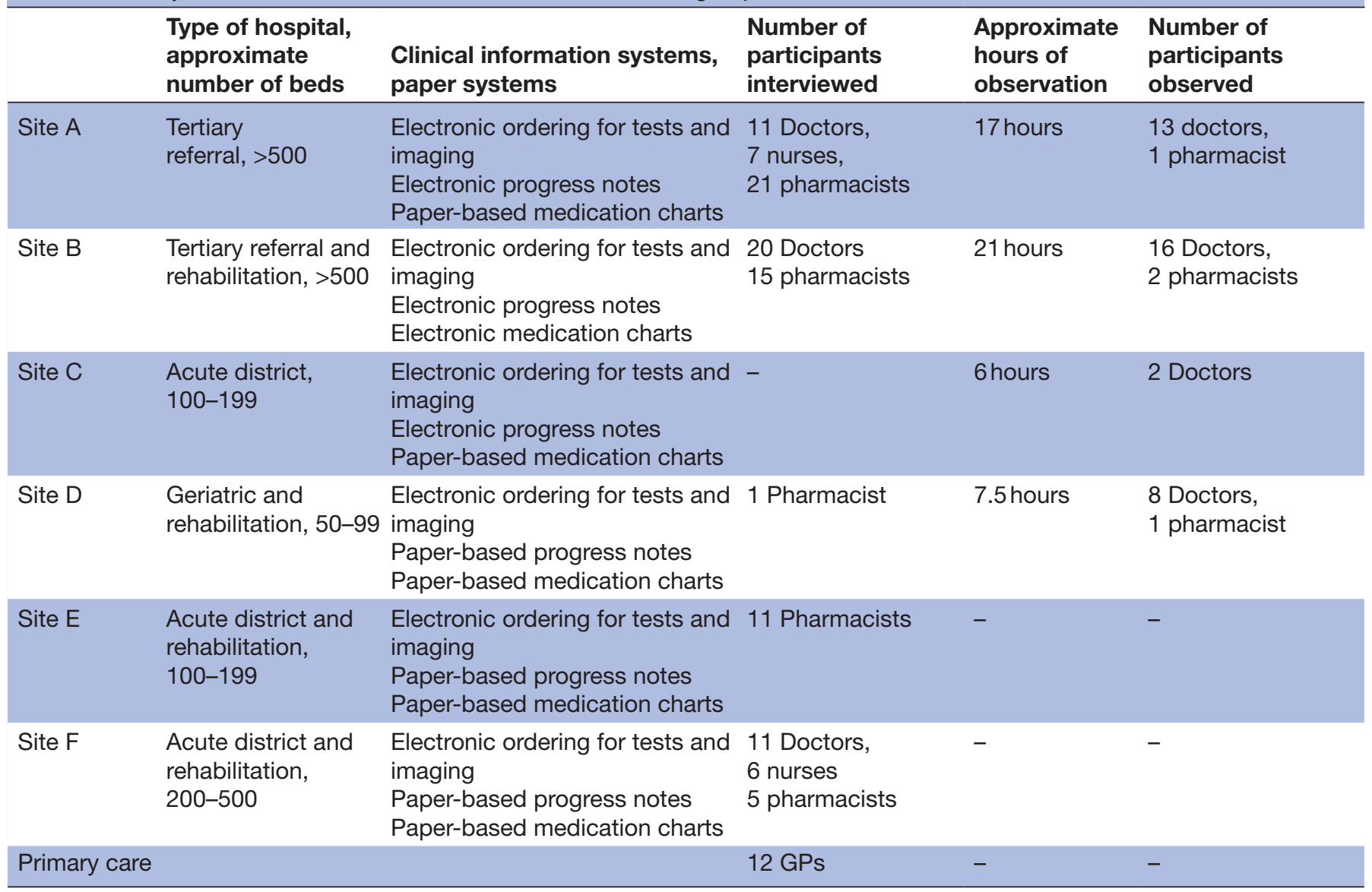

Some participants took part in both observations and interviews.

researchers with expertise in qualitative research, met periodically throughout data collection to discuss and compare identified themes. Any disagreements in themes were discussed until a consensus was reached.

\section{Public and patient involvement statement}

No patients or the public were involved in any stage of the research process for this study.

\section{Ethics approval}

Written informed consent was obtained from all participants in the study.

\section{RESULTS}

\section{Medicines review-the current process}

In total, 117 instances of medicines review were observed across four hospitals. Table 2 summarises the data collected from observations.

During interviews and focus groups with hospital staff, participants demonstrated an inconsistent understanding of what medicines review was and of what it involved. Participants spoke about obtaining a best possible medication history, undertaking medication reconciliation and reviewing inpatient medication charts.
A pharmacist said: By med review, are you talking about the first med review, when you're doing the history and the whole full thing or are you talking about a daily or every two days, whatever you might do, have a new order, check the new antibiotic and see if it's appropriate, are there any interactions? That also can be called a med review. (P1013)

And a doctor: I think it's a little undefined in terms of who does it, and depending on the time of the day, even the day of the week, you'll have different people doing it. (D4004)

Most participants explained that a medicines review was undertaken within a few days of admission and triggered at certain time points in a patient's journey, such as transfer between settings (eg, emergency department to the ward) and at discharge from hospital. This is consistent with what we observed on wards (see table 2). During discussions with staff, it became apparent that medicines review was perceived to be the role of doctors, both senior and junior, and of pharmacists.

Participants reported that common triggers for medicines review were patient factors (eg, age, comorbidities), medication-related factors (eg, high risk medicines) and requests from other healthcare providers.

The information that participants reported accessing during medicines review depended on their understanding of the process. For example, participants noted 
Table 2 Characteristics of medicines reviews and episodes of deprescribing observed during 52 hours of observations across four Sydney hospitals

\begin{tabular}{|c|c|c|c|c|c|}
\hline \multirow{3}{*}{ Medicine reviews } & & Site A & Site B & Site C & Site D \\
\hline & & \multicolumn{4}{|c|}{ Number of times observed } \\
\hline & & 39 & 45 & 12 & 21 \\
\hline \multirow[b]{3}{*}{ Type of medicine review } & Initial review & 5 & 9 & 1 & 3 \\
\hline & Follow-up & 23 & 30 & 5 & 14 \\
\hline & Discharge & 11 & 6 & 6 & 4 \\
\hline \multirow[b]{3}{*}{ Location of medicine review* } & Hallway & 16 & 19 & 9 & 7 \\
\hline & Patient bedside & 19 & 31 & 8 & 10 \\
\hline & Other (eg, office) & 7 & 6 & 2 & 5 \\
\hline \multirow[b]{3}{*}{ Tyne of device used* } & cow & 26 & 37 & 10 & 5 \\
\hline & Computer at $\mathrm{JMO} /$ registrar workstation & 3 & 3 & 2 & 2 \\
\hline & Computer at pharmacy workstation & - & 3 & - & 1 \\
\hline \multicolumn{2}{|l|}{$\begin{array}{l}\text { Medicine reviews where } \\
\text { deprescribing occurred }\end{array}$} & $15(38 \%)$ & $24(53 \%)$ & $4(33 \%)$ & $7(33 \%)$ \\
\hline \multicolumn{6}{|l|}{ In deprescribing cases: } \\
\hline \multirow{4}{*}{$\begin{array}{l}\text { Provider who requested the } \\
\text { change to a medication }\end{array}$} & Consultant & 11 & 11 & 1 & 6 \\
\hline & Registrar & 3 & 10 & 3 & 1 \\
\hline & $\mathrm{JMO}$ & - & 3 & - & - \\
\hline & Pharmacist & 1 & - & - & - \\
\hline \multirow{3}{*}{$\begin{array}{l}\text { Provider who changed the } \\
\text { medication }\end{array}$} & Consultant & - & 2 & - & - \\
\hline & Registrar & 6 & 7 & 4 & 5 \\
\hline & JMO & 9 & 15 & - & 2 \\
\hline \multirow{10}{*}{$\begin{array}{l}\text { Artefacts accessed } \\
\text { Italicised artefacts are those } \\
\text { accessed via the electronic } \\
\text { medical record }\end{array}$} & Paper medication chart & 11 & - & 4 & 6 \\
\hline & Patient list & 4 & 12 & 1 & 1 \\
\hline & Observations & - & - & 2 & 1 \\
\hline & Progress notes & 13 & 14 & 3 & - \\
\hline & Test results & 8 & 18 & 3 & 2 \\
\hline & Medication list & - & 8 & - & - \\
\hline & MAR/MAR Summary & - & 20 & - & - \\
\hline & Orders (medications, pathology) & 6 & 5 & 2 & - \\
\hline & Observations & 4 & 6 & - & - \\
\hline & $\begin{array}{l}\text { External resources (CIAP to access } \\
\text { AMH, MIMS, eTG) }\end{array}$ & 1 & 5 & 1 & - \\
\hline
\end{tabular}

*Numbers are greater than total events observed as single events could have included multiple locations, devices and artefacts. AMH, Australian Medicines Handbook; CIAP, Clinical Information Access Portal; COW, computer on wheels; JMO, juniormedical officer; MAR, Medication Administration Record; MIMS, Monthly Index of Medical Specialties, eTG, Electronic Therapeutic Guidelines.

that key information sources for medication reconciliation were GPs, patients, families, residential aged care facilities and pharmacies. Participants who viewed medicines review as a review of the inpatient medication chart described accessing inpatient notes, medications and pathology results. This is consistent with what we observed (table 2).

\section{Barriers to medicines review}

The main barrier reported to impact on medicines review was limited time.
A pharmacist said: Obviously, ideally, you would want to do that medication review after you do that history taking. But then, you have so many patients that come in that all need history taking... we just don't have time. (P2004)

Other reported barriers were short length of stay (preventing a detailed review) and missing or unreliable information in clinical information systems.

Awareness and understanding of polypharmacy

Participants appeared to have a strong understanding of polypharmacy, with many emphasising that it is not 
simply the number of medications that a patient is taking, but also whether the medications are needed. Clinicians discussed the concept of inappropriate polypharmacy for the individual patient.

A doctor said: I think of polypharmacy probably more as contextual in the sense of what can the patient manage and what are the indications. (D1026)

And a pharmacist: 'Irrational medications' is a good definition because some patients would require a lot of medications, much more than five, but if they're rational, that's fine. If they're just sort of added on and never reviewed, then that comes into my definition of polypharmacy. (P1010)

A nurse explained: The person will be prescribed a new medication and they may have some side effects from the medication, but without investigating that, they're given more medication to treat the side effects. So that's the cascade effect. And a lot of them aren't necessary, so that's why we should be deprescribing. (N1008)

\section{Deprescribing-the current process}

Participants viewed deprescribing as the process of rationalising a patient's medications. That is, stopping or reducing the dose of medications when the harms outweigh the benefits or when medications are no longer needed.

A doctor said: Considering the risk benefit profile of that medication, so it might have an indication, but does the indication outweigh the risk? (D4015)

Deprescribing was reported to occur during medicines review, as an outcome of the review. Participants reported that registrars and consultants were primarily responsible for deprescribing, although junior doctors did deprescribe medications where it was clear to them that there was no current indication (eg, common electrolytes, antihypertensives, proton pump inhibitors and analgesics) and/or medications that were causing adverse effects in the patient. A junior doctor explained: It's kind of difficult because in that situation it's like we have no agency, like the consultant comes in we're gonna slash this, this, and this. (D4039)

A pharmacist said: They're [JMOs are] not the ones making that decision, but they are the ones that enter it. (P5001)

Data obtained from observations were consistent with these views. As shown in table 2, senior doctors, including consultants and registrars, were observed to make the deprescribing decisions (in 92\% of deprescribing episodes), although it was the junior doctors who made many of the changes to medication orders. Consultants were observed to manually change a medication order only in two instances.

\section{Barriers to deprescribing}

A large number of barriers to deprescribing were identified via our interviews and focus groups with hospital staff. Although some senior geriatricians viewed deprescribing to be a central part of their role, a key barrier that emerged from interviews was that hospital clinicians, particularly junior staff, did not view deprescribing to be their responsibility. They viewed their primary responsibility to be treating the acute health problem(s), not patients' chronic health conditions. Some participants were explicit in saying that they believed it was the GP's responsibility to deprescribe but not that of the hospital doctor.

A hospital doctor said: Well, I rarely stop patient's medicines...I don't think I do-unless, it's contraindicated or something, I would never really deprescribe because maybe the GP has put them on it for a particular reason. (D1063)

A large number of pharmacists also explained that they thought hospital doctors were reluctant to deprescribe:

And the doctors here are reluctant to make any changes or to stop anything because they said, 'If the GP started it, there probably is a reason why the GP started it', and so they go back into the community with those same medications that they came in on plus the additional ones that have been started. (P3006)

Some hospital participants, especially more junior or less experienced staff, discussed fear or insecurity associated with deprescribing.

A junior doctor explained: As a JMO, I would feel uncomfortable deprescribing unless I ran it pass the registrar first and make sure they were happy. (D4040)

And a pharmacist: It really depends on the confidence of that registrar in making that decision, the clinical decision. Because they really vary in how they feel about making the call. And I think I sense a lot of fear about deprescribing. (P3009)

Some pharmacists and GPs indicated that a patient's hospital stay was an ideal time to deprescribe. However, GPs also recognised that in-hospital deprescribing was likely to be dependent on the reason for a patient's admission and the team caring for a patient.

Another key barrier to deprescribing identified was patients, particularly when excluded from or not engaged in the deprescribing decision.

A pharmacist said: I just feel sad for the patient and I think we need to bring them more into the discussion and get them on board because otherwise they're just going to go home and keep taking them. (P3003)

And a doctor explained: When they [patients] go home, if you haven't counselled, educated, whatever, they don't know you changed things, they're just going to do whatever they want, they'll just revert to their normal medications. So that engagement of the patient in the deprescribing is very important... (D2012)

GPs also highlighted the important role patients play in deprescribing.

The patient's got to understand why and how it's [deprescribing] going to happen, and that they're going to have a sort of collaborative relationship with the GP for them to get rid of stuff and monitor for side effects, monitor for interactions. (GP7003)

Other barriers to deprescribing included not being able to contact the initial prescriber and complexity of deprescribing. Hospital doctors explained that they were reluctant to deprescribe when deprescribing was not straightforward (eg, if weaning of the medication was complex, or it would be difficult to determine the side effects of deprescribing). 


\section{Communication of deprescribing decisions}

Communication of deprescribing was reported to be primarily via the discharge summary. Hospital clinicians said that it was important to clearly communicate what is being stopped, why (including the current indication) and how (including time frame). Any in-hospital deprescribing was unlikely to be successful or sustained if not communicated effectively to the GP and to the patient. This was consistent with what was valued by GPs. GPs viewed communication of the reasons why medications were changed or ceased by clinicians in hospital to be critical for them to continue the deprescribing process.

A GP explained: If they said the reason why certain medications are being altered or amended or stopped or started, with a reason and then sometimes there's a follow up action... so recommend to review it in $X$ period of time or to check their blood pressure in a certain period of time, that's the most useful because you can completely see the perspective having not seen what happened in the hospital. You can get a window into the rationale behind $i$, and then clinically you're able to follow up with a sensible plan. (GP7001)

Some GPs also expressed a preference for receiving direct communication for complex patient cases.

GPs felt that effective communication of in-hospital deprescribing to GPs would facilitate continuity of care. If well-informed, GPs with strong, long-standing relationships with patients would then be able to communicate medication changes to their patients, ensuring that patients are aware of how and why changes had been made.

\section{Preferences for EDS}

Table 3 summarises the main preferences expressed by participants with respect to the content, form and recipients of EDS.

\section{Content and format of decision support}

When doctors, nurses and pharmacists were asked about EDS to facilitate deprescribing in older inpatients, their responses were highly variable. A frequent suggestion was guidelines on how to effectively deprescribe potentially inappropriate medicines, with clear rationale for deprescribing, including advice on who to consult and how to communicate deprescribing decisions. Participants emphasised that guidance on what to do (ie, actions to take) needed to be accompanied by strong evidence for why those actions were needed. This would provide pharmacists, nurses and more junior medical staff with material to support deprescribing when consulting with senior clinicians.

Regarding the form EDS should take, clinicians were adamant that alerts would not be effective because too many alerts would result in alert fatigue. Almost all participants were opposed to the idea of using alerts to indicate when deprescribing may be appropriate. Instead, participants liked the idea of a traffic light system, where colour

Table 3 User preferences for electronic decision support

\begin{tabular}{|c|c|c|}
\hline & Theme & Illustrative quotes \\
\hline $\begin{array}{l}\text { Content of } \\
\text { EDS }\end{array}$ & $\begin{array}{l}\text { Why and how to } \\
\text { deprescribe }\end{array}$ & $\begin{array}{l}\text { An evidence-based sort of way of deprescribing or dose reduction, yeah definitely. (Doctor } \\
4040 \text { ) }\end{array}$ \\
\hline \multirow[t]{3}{*}{$\begin{array}{l}\text { Form of } \\
\text { EDS }\end{array}$} & $\begin{array}{l}\text { Electronic } \\
\text { guidelines }\end{array}$ & $\begin{array}{l}\text { How to do the deprescribing... a guideline... where you can quickly just look up and then see } \\
\text { if the person's yes or no. (Pharmacist 3007) }\end{array}$ \\
\hline & & $\begin{array}{l}\text { The tricky thing with alerts is there's definitely that sense of alert fatigue where you're just } \\
\text { like, 'Oh I'm sick of this. So close, nah. I'm not even following you out of spite.' (Doctor 4002) }\end{array}$ \\
\hline & Traffic light system & I think colour's a nice idea. (Doctor 4012) \\
\hline \multirow{3}{*}{$\begin{array}{l}\text { Recipients } \\
\text { of EDS }\end{array}$} & Senior doctors & $\begin{array}{l}\text { But also getting the right target. So I think it's the consultants and the registrars making } \\
\text { a lot of the decisions. So, hounding the intern continuously with alerts, they may pass on } \\
\text { the message? Maybe targeting the people who are more likely to make the deprescribing } \\
\text { decision. (Doctor 4004) }\end{array}$ \\
\hline & $\begin{array}{l}\text { Users of the } \\
\text { electronic system }\end{array}$ & $\begin{array}{l}\text { I suppose the JMOs do most of the prescribing. Well the actual typing it out and all, hand } \\
\text { writing stuff. They're probably your biggest people to target. (Pharmacist 5001) }\end{array}$ \\
\hline & $\begin{array}{l}\text { Those with limited } \\
\text { knowledge }\end{array}$ & $\begin{array}{l}\text { And that's where I think it's like a grammar and spell check. I mean if you're confident, you } \\
\text { don't use it. You just go on, that's great, I don't need anyone to check my spelling. But if } \\
\text { you're not, then you use something. (Doctor 2009) }\end{array}$ \\
\hline
\end{tabular}


is used to indicate risk of adverse outcomes due to polypharmacy. Participants expressed a preference for short, simple advice that is easy to access (ie, a few clicks away) and integrates well into current workflows.

\section{Recipients of decision support}

Many participants, particularly doctors, indicated a preference for directing the EDS to pharmacists. Clinicians felt that this would result in more of the information being reviewed. Pharmacists could then communicate key potential harms and benefits to doctors. Related to this, participants believed that communication of advice in person was likely to be more effective than communication via a computer.

With respect to doctors as the recipients of EDS, participants were divided, with some indicating that junior doctors should be the target, and others indicating that it should be more senior doctors.

One pharmacist questioned the value of designing decision support embedded in the electronic system, as this would not target the senior clinicians and ultimately render the decision support ineffective. In terms of the decision being made on the patient, you're targeting the lowest kind of person who has the least authority for making decisions for the patient, by making a decision support tool that sits within eMR [electronic medical record]. (P1018)

Other participants stressed the importance of directing decision support to those with limited knowledge (ie, those working outside of geriatrics).

\section{DISCUSSION}

Wide engagement with hospital doctors, nurses and pharmacists revealed variable understanding of medicines review and what this process involved. Despite this, our observational data confirmed that all clinicians undertook medicines review and were fairly consistent in how this was performed. Polypharmacy and deprescribing were well understood by all participants. The act of deprescribing was typically performed by junior doctors and occurred during medicines review under the instruction of senior doctors. We identified a number of barriers to deprescribing, primarily a perception that deprescribing was not the responsibility of hospital doctors, a lack of confidence among junior doctors and pharmacists in broaching this topic with senior doctors and a lack of patient engagement in the deprescribing process. Hospital staff and GPs reported that with improved communication between hospitals and GPs on how and why deprescribing occurred, these decisions were more likely to be sustained.

In consolidating our results, we aimed to inform the design of EDS by determining the right information to provide, the right time to provide it and the right person to target. ${ }^{9}$ As deprescribing decisions were made at multiple time points throughout a patient's admission, particularly during a patient's initial medicines review, the EDS would need to be available at all times or 'on-demand' to accommodate various clinical workflows. As decision support would be embedded within the electronic medical record, it follows that the recipients of the EDS would be the users of the electronic system. Our observations confirmed that junior doctors, pharmacists and nurses used the system to a much greater extent than the primary decision-makers (senior doctors), a finding consistent with other research. ${ }^{13}$ Thus, the EDS would need to be informative and relevant so as to motivate users to initiate a conversation with the senior clinician. Junior doctors, pharmacists and nurses expressed a strong preference for guidance on not only how to deprescribe but also why, this latter information required to support deprescribing when consulting with senior doctors. Finally, EDS should facilitate communication of information about in-hospital deprescribing, including what medications were changed and why, to patients' primary care providers.

We found strong resistance among users to the implementation of interruptive alerts to signal patient risk or polypharmacy. Alerts are frequently used form of decision support but are often not read. ${ }^{18}$ Alert fatigue, a consequence of too many false-positive alerts being triggered, is a common and persistent problem. ${ }^{19}$ Opposition to the inclusion of alerts in our study is therefore not surprising and is consistent with previous reviews of EDS for deprescribing, which highlight the challenges of using alerts. ${ }^{1011}$

The barriers to deprescribing we identified in our study are in-line with those reported in previous research, including a diffusion of responsibility and low self-efficacy (ie, confidence in knowing when to approach senior staff). ${ }^{14-1620}$ In overcoming these barriers, EDS is likely to represent only one component of a multifaceted intervention. EDS can prompt users when patients are at risk of polypharmacy and subsequently provide junior doctors, nurses and pharmacists with evidence to alert and influence the senior clinicians, increasing perceived competence and self-confidence. However, to shift perceptions of roles and responsibilities, it is likely that an additional approach will be needed. For example, the Behaviour Change Wheel, a well-known framework of behaviour change interventions, ${ }^{21}$ suggests that beliefs about professional role (ie, motivation) are influenced by training and by policy change such as regulation. Interestingly, Australia's updated standards for hospital accreditation include in-hospital medication review and shared decision making with consumers. ${ }^{22}$

A key result that emerged from our study was the vital role that patients play in deprescribing. Both hospital staff and GPs emphasised the importance of engaging patients in the process in order to ensure continuity of care and prevent represcribing. The benefits of shared decision making are well known, ${ }^{23} 24$ although research has suggested that it is not often practiced. ${ }^{25}$ EDS can facilitate patient engagement in deprescribing to some extent by, for example, automatically populating deprescribing decisions into a patient's discharge summary, 
providing GPs with the critical information to reiterate and reinforce decisions made in hospital to the patient. In addition, communication between in-hospital clinicians and patients on rationale, expectations and preferences is likely to result in a more effective approach than EDS alone, in ensuring deprescribing decisions are understood, agreed with, and sustained, which is a focus of our ongoing research.

\section{Strengths and weaknesses of the study}

This study comprised an in-depth investigation of medicines review and deprescribing with a large number of multidisciplinary clinicians. By complementing interviews with in situ observation, we were able to validate some participants' perceptions with objective data, although we acknowledge that our results are primarily drawn from interviews and may be subject to biases associated with self-report. The perceptions and practices described are likely to be representative of geriatric medicine, general medicine and rehabilitation wards in metropolitan Sydney teaching hospitals and may not be generalisable to other clinical services or different settings. In particular, while addressing the patient's chronic disease management (which includes medication review) is considered part of standard comprehensive geriatric assessment and best practice for older adults admitted to hospital, ${ }^{26}$ this may not be the standard model of care for other hospital specialties or primary care settings such as nursing homes.

\section{CONCLUSION}

Deprescribing, undertaken during medicines review, is often performed by a junior doctor, following a decision to deprescribe by a senior doctor. In designing effective decision support for deprescribing in the electronic medical record, the EDS, likely to be used by junior doctors, pharmacists and nurses, should be available throughout the hospitalisation and should comprise non-interruptive evidence-based guidance on why and how to deprescribe. Deprescribing decisions are complex and influenced by multiple factors. Thus, the implementation of EDS alone may not result in sustained improvements in review of polypharmacy and subsequent deprescribing. Our future work will focus on developing and refining EDS design, while developing complementary interventions with clinicians and patients.

\section{Author affiliations}

${ }^{1}$ Faculty of Health Sciences, The University of Sydney, Sydney, New South Wales, Australia

${ }^{2}$ Centre for Health Systems \& Safety Research, Australian Institute of Health Innovation, Macquarie University, Sydney, New South Wales, Australia ${ }^{3}$ Department of Clinical Pharmacology and Aged Care, Kolling Institute of Medical Research, Royal North Shore Hospital School, Saint Leonards, New South Wales, Australia

${ }^{4}$ St Vincent's Clinical School, University of New South Wales, Sydney, New South Wales, Australia

${ }^{5}$ Department of Clinical Pharmacology and Aged Care, Royal North Shore Hospital School, Saint Leonards, New South Wales, Australia

${ }^{6}$ Capital and Coast District Health Board, Wellington, New Zealand
${ }^{7}$ Health Informatics Unit, Sydney Local Health District, Camperdown, New South Wales, Australia

${ }^{8}$ Concord Clinical School, The University of Sydney, Sydney, New South Wales, Australia

${ }^{9}$ University of Sydney Centre for Education and Research on Ageing, Concord, New South Wales, Australia

${ }^{10}$ Faculty of Pharmacy, The University of Sydney, Sydney, New South Wales, Australia

${ }^{11}$ NSW Therapeutic Advisory Group, Darlinghurst, Australia

${ }^{12}$ Northern Clinical School, The University of Sydney, Sydney, New South Wales, Australia

Acknowledgements We would like to acknowledge the contribution of Northern Sydney Primary Health Network in recruiting general practitioners for this study.

Contributors SH, MTB, DLC, AM and AB all made contributions to study conception and design. MD, AN, SL and BN undertook data collection. MTB, MD, AN and WYZ undertook data analysis. All authors contributed to data interpretation, particularly AR who provided input with respect to the electronic system. All authors contributed to and approved the final manuscript.

Funding This research was supported by a NSW Health Translational Research Grant Scheme Grant.

Competing interests None declared.

Patient consent for publication Not required.

Ethics approval Ethics approval was obtained from Northern Sydney Local Health District Human Research Ethics Committee (LNR/17/HAWKE/138), with site-specific approval obtained from each participating hospital.

Provenance and peer review Not commissioned; externally peer reviewed.

Data sharing statement Non-identifiable data are available on request from authors.

Open access This is an open access article distributed in accordance with the Creative Commons Attribution Non Commercial (CC BY-NC 4.0) license, which permits others to distribute, remix, adapt, build upon this work non-commercially, and license their derivative works on different terms, provided the original work is properly cited, appropriate credit is given, any changes made indicated, and the use is non-commercial. See: http://creativecommons.org/licenses/by-nc/4.0/.

ORCID iD

Melissa T Baysari http://orcid.org/0000-0003-1645-9126

\section{REFERENCES}

1. Gallagher P, Lang PO, Cherubini A, et al. Prevalence of potentially inappropriate prescribing in an acutely ill population of older patients admitted to six European hospitals. Eur J Clin Pharmacol 2011;67:1175-88.

2. Hajjar ER, Hanlon JT, Sloane RJ, et al. Unnecessary drug use in frail older people at hospital discharge. J Am Geriatr Soc 2005;53:1518-23.

3. Donaldson LJ, Kelley ET, Dhingra-Kumar N, et al. Medication without harm: WHO's Third Global Patient Safety Challenge. Lancet 2017;389:1680-1.

4. Reeve E, Shakib S, Hendrix I, et al. Review of deprescribing processes and development of an evidence-based, patient-centred deprescribing process. Br J Clin Pharmacol 2014;78:738-47.

5. Tannenbaum C, Farrell B, Shaw J, et al. An ecological approach to reducing potentially inappropriate medication use: Canadian Deprescribing Network. Can J Aging 2017;36:97-107.

6. Stewart D, Mair A, Wilson M, et al. Guidance to manage inappropriate polypharmacy in older people: systematic review and future developments. Expert Opin Drug Saf 2017;16:203-13.

7. Garg AX, Adhikari NK, McDonald H, et al. Effects of computerized clinical decision support systems on practitioner performance and patient outcomes: a systematic review. JAMA 2005;293:1223-38.

8. Schedlbauer A, Prasad V, Mulvaney C, et al. What evidence supports the use of computerized alerts and prompts to improve clinicians' prescribing behavior? J Am Med Inform Assoc 2009;16:531-8.

9. Wright A, Phansalkar S, Bloomrosen M, et al. Best practices in clinical decision support. Appl Clin Inform 2010;1:331-45.

10. Dalton K, O'Brien G, O'Mahony D, et al. Computerised interventions designed to reduce potentially inappropriate prescribing in 
hospitalised older adults: a systematic review and meta-analysis. Age Ageing 2018;47:670-8.

11. Scott IA, Pillans PI, Barras M, et al. Using EMR-enabled computerized decision support systems to reduce prescribing of potentially inappropriate medications: a narrative review. Ther $\mathrm{Adv}$ Drug Saf 2018;9:559-73.

12. van der Sijs $\mathrm{H}$, Aarts J, Vulto $\mathrm{A}$, et al. Overriding of drug safety alerts in computerized physician order entry. J Am Med Inform Assoc 2006;13:138-47.

13. Baysari MT, Westbrook Jl, Richardson KL, et al. The influence of computerized decision support on prescribing during ward-rounds: are the decision-makers targeted? J Am Med Inform Assoc 2011;18:754-9.

14. Anderson K, Stowasser D, Freeman C, et al. Prescriber barriers and enablers to minimising potentially inappropriate medications in adults: a systematic review and thematic synthesis. BMJ Open 2014;4:e006544.

15. Jubraj B, Marvin V, Poots AJ, et al. A pilot survey of junior doctors' attitudes and awareness around medication review: time to change our educational approach? Eur J Hosp Pharm 2015;22:243-8.

16. Reeve E, Thompson W, Farrell B. Deprescribing: a narrative review of the evidence and practical recommendations for recognizing opportunities and taking action. Eur J Intern Med 2017;38:3-11.

17. Thomas DR. A general inductive approach for analyzing qualitative evaluation data. Am J Eval 2006;27:237-46.

18. Baysari MT, Westbrook JI, Richardson $\mathrm{K}$, et al. Optimising computerised alerts within electronic medication management systems: a synthesis of four years of research. Stud Health Technol Inform 2014;204:1-6.

19. Payne TH. EHR-related alert fatigue: minimal progress to date, but much more can be done. BMJ Qual Saf 2019;28:1-2.

20. Kouladjian L, Gnjidic D, Reeve E, et al. Health care practitioners' perspectives on deprescribing anticholinergic and sedative medications in older adults. Ann Pharmacother 2016;50:625-36.

21. Michie S, van Stralen MM, West R. The behaviour change wheel: a new method for characterising and designing behaviour change interventions. Implement Sci 2011;6:42.

22. Australian Commission on Safety and Quality in Health Care. National Safety and Quality Health Service Standards. Sydney: ACSQHC, 2017.

23. Stiggelbout AM, Van der Weijden T, De Wit MP, et al. Shared decision making: really putting patients at the centre of healthcare. $B M J$ 2012;344:e256.

24. Jansen J, Naganathan V, Carter SM, et al. Too much medicine in older people? Deprescribing through shared decision making. BMJ 2016;353:i2893.

25. Baysari M, Westbrook J, Day R. Medication decision-making on hospital ward-rounds. Stud Health Technol Inform 2011;169:935-9.

26. Ellis G, Gardner M, Tsiachristas A, et al. Comprehensive geriatric assessment for older adults admitted to hospital. Cochrane Database Syst Rev 2017;9:Cd006211. 